\title{
Adaptive scheduling for millimeter wave multi-beam satellite communication systems
}

\author{
ZHOU Zhengyi ${ }^{1}$, FENG Wei ${ }^{1}$, CHEN Yunfei ${ }^{2}$, GE Ning ${ }^{1}$ \\ 1. Tsinghua National Laboratory for Information Science and Technology Department of Electronic Engineering, Tsinghua University, \\ Beijing 100084, China \\ 2. School of Engineering, University of Warwick, Coventry CV4 7AL, UK
}

\begin{abstract}
In space information networks, the performance of the satellite-terrestrial links is crucial for downlink data delivery. Due to their relatively wide bandwidth, the use of mmWave (millimeter wave) signals is promising to enhance satellite communications. Adopting on-board antenna arrays can mitigate the attenuation associated with mmWave signals, and can also enable multi-beam transmissions. However, the number of RF (Radio Frequency) chains is usually limited in practical applications, which makes it extremely difficult to properly schedule users. In general, this scheduling problem is NP-hard. In this paper, we propose two scheduling algorithms that can be used to solve this problem with relatively low computational complexity. Intuitively, the correlated users are separated by the proposed schemes, which implies that the spatial diversity gain of multiple beams can be exploited. Simulation results show that the proposed algorithms can achieve nearly optimal performance in terms of the achievable sum rate of the system.
\end{abstract}

Key words: millimeter wave, satellite, scheduling, beamforming, antenna arrays

\section{Introduction}

Rapidly increasing demand for higher data rates has become a challenging problem for next generation satellite communication systems, and one of the most significant constraints is the shortage of available bandwidth. To address this situation, either new spectrum must be made available, or higher spectral efficiency must be achieved.

The mmWave band promises to offer abundant spectral resources ${ }^{[1,2]}$. Satellite communications in the $\mathrm{Ka}$ band and above have been investigated $\mathrm{d}^{[3-5]}$, and
mmWave channels have been found to be generally LoS (Line-of-Sight)-dominated because mmWave signals suffer from severe attenuation ${ }^{[6]}$. With much smaller wavelengths, mmWave technology enables a large number of antennas in a given area. Antenna arrays driven by smart beam selection algorithms can be utilized to mitigate mmWave signal attenuation ${ }^{[6]}$. The deterioration of system performance under LOSdominated channel conditions can be significantly alleviated by directed beamforming. The hybrid beamforming scheme shown in Fig.1 further ensures a feasible implementation framework for large arrays

Manuscript received May 16, 2016; accepted Jul. 28, 2016

This work is supported by the National Natural Science Foundation of China (Nos.61132002, 61321061). 
by reducing the number of RF chains ${ }^{[7]}$. Channel estimation methods and precoding algorithms are also investigated in detail in Refs.[7-12].

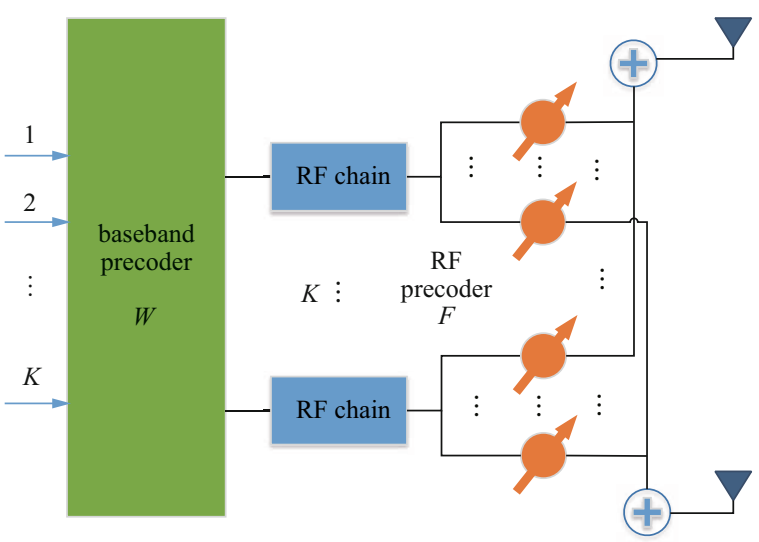

Figure 1 Implementation of hybrid precoding

In order to provide reasonable system capacity, the number of active users in the system should be no more than the number of RF chains ${ }^{[13]}$. This can be addressed by network densification in terrestrial wireless communications. However, scheduling is inevitable in satellite communications systems, as shown in Fig.2. It is not wise to arbitrarily choose active users from among all the users to be served because serving nearby directional users simultaneously is extremely challenging for beamforming ${ }^{[14]}$. As a result, the quality of service (QoS) is heavily degraded no matter what precoding algorithm is adopted. The ideal scheduling method

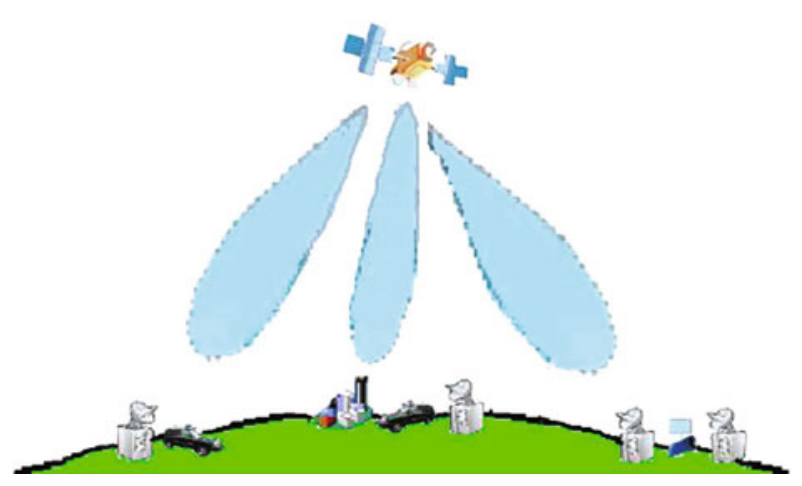

Figure 2 Satellite systems with mmWave hybrid beamforming arrays divides closely located users into different time slots, and ensures active users in the same time slot can be easily distinguished in order to obtain spatial multiplex gain.

This paper focuses on the scheduling problems in satellite communication systems that use mmWave hybrid beamforming antenna arrays. The scheduling model is formulated in Section 2, scheduling algorithms are proposed in Section 3, simulation results are shown in Section 4, and the paper is concluded in Section 5.

\section{Problem formulation}

We assume that there are a total of $N$ users in need of service within our satellite communication system, which has a channel matrix $\boldsymbol{H} \in \mathbb{C}^{N \times M}$, where $M$ is the size of the satellite antenna arrays, and $K$ $(K<N) \mathrm{RF}$ chains are available due to practical implementation constraints. This means that the satellite is capable of serving at most $K$ users in one time slot, assuming that each user requires one independent data stream. The $N$ users are divided into $G$ groups with $K_{1}, K_{2}, \cdots, K_{G}$ users in each group and corresponding channel sub-matrix $\boldsymbol{H}_{1}, \boldsymbol{H}_{2}, \cdots, \boldsymbol{H}_{G}$ respectively. It takes $G$ time slots in the aggregate to serve all $G$ groups of users.

For simplicity, we let $G=N / K$ be an integer, and create an average partition, as follows.

$$
K_{1}=K_{2}=\cdots=K_{G}=K .
$$

In time slot $m$, we adopt the mmWave uniform rectangular array (URA), and perform hybrid beamforming to serve the $K$ users with channel matrix $\boldsymbol{H}_{m}$. The hybrid precoding model is stated in detail in Refs. $[7,8,10]$. If the transmitted symbol vector is denoted as $\boldsymbol{x}$, the received symbol of the $K$ users together can be expressed as

$$
\boldsymbol{y}=\rho \beta \boldsymbol{H}_{m} \boldsymbol{F}_{m} \boldsymbol{W}_{m} \boldsymbol{x}+n,
$$

where $\rho$ is the multiplier for antenna gain, $\beta$ is the 
multiplier for pathloss, and $\boldsymbol{F}_{m} \in \mathbb{C}^{M \times K}$ is the analog precoding matrix satisfying

$$
\left[\boldsymbol{F}_{m}\right]_{i, j}=\frac{1}{\sqrt{M}} \mathrm{e}^{\mathrm{j} 2 \pi \phi_{i, j}},
$$

$\boldsymbol{W}_{m}=\left[\boldsymbol{w}_{m, 1}, \boldsymbol{w}_{m, 2}, \cdots, \boldsymbol{w}_{m, K}\right] \in \mathbb{C}^{K \times K}$ is the baseband digital precoding matrix. $n \in \mathbb{C}^{K \times 1}$ is the complex additive white Gaussian noise vector with diagonal covariance matrix. $\boldsymbol{x}$ satisfying

$$
\mathrm{E}\left[x x^{\mathrm{H}}\right]=\frac{P}{K} I_{K},
$$

where $P$ is the total transmit power. The digital precoder $\boldsymbol{W}_{m}$ is normalized to meet the total power constraint

$$
\left|\boldsymbol{F}_{m} \boldsymbol{W}_{m}\right|_{F}^{2}=K .
$$

The clustered mmWave channel model characterizing the limited scattering feature ${ }^{[7,8,10,11,15,16]}$ is employed here for the downlink channel matrix of the $K$ users in the group $H_{m}=\left[h_{m, 1}, h_{m, 2}, \cdots, h_{m, K}\right]^{\mathrm{T}} \in \mathbb{C}^{K \times M}$. The column $h_{m, k}$ is the channel vector of the $k$ th user in the $m$ th group. Taking into account the LoS-dominated characteristics and the attenuation of the mmWave signals, we employ two paths for the channel model including the LoS path.

$$
\boldsymbol{h}_{m, k}=\sqrt{\frac{M}{2}} \sum_{i=1}^{2} \alpha_{m, k}^{i} \boldsymbol{a}\left(\phi_{m, k}^{i}, \theta_{m, k}^{i}\right),
$$

where $\alpha_{m, k}^{1} \sim \mathcal{C N}(0,1), \alpha_{m, k}^{2} \sim \mathcal{C N}\left(0, \sigma^{2}\right)$ are the complex gains of the paths with the Rician factor $\sigma^{2} . \phi_{m, k}^{i}\left(\theta_{m, k}^{i}\right)$ is the azimuth (elevation) angle of the corresponding user, and $\boldsymbol{a}\left(\phi_{m, k}^{i}, \theta_{m, k}^{i}\right)$ is the antenna array response vector determined by the geometric architecture of the arrays. For URA,

$$
\begin{aligned}
\boldsymbol{a}(\phi, \theta)= & \frac{1}{\sqrt{M}}\left[1, \cdots, \mathrm{e}^{\mathrm{j} \frac{2 \pi}{\lambda} d(m \sin \phi \sin \theta+n \cos \theta)}, \cdots,\right. \\
& \left.\mathrm{e}^{\mathrm{j} \frac{2 \pi}{\lambda} d\left(\left(N_{h}-1\right) \sin \phi \sin \theta+\left(N_{v}-1\right) \cos \theta\right)}\right]^{\mathrm{T}},
\end{aligned}
$$

where $N_{v}\left(N_{h}\right)$ is the number of antennas in the vertical (horizontal) direction within the arrays $\left(N_{h} N_{v}=M\right)$. $m, n$ are the indices of each antenna element,
$0 \leqslant m \leqslant\left(N_{h}-1\right), 0 \leqslant n \leqslant\left(N_{v}-1\right)$.

The SINR (Signal-to-Interference-plus-Noise Ratio) of the $k$-th user in the $m$-th group can be expressed as

$$
\operatorname{SINR}_{m, k}=\frac{\frac{\rho \beta P}{K}\left|\boldsymbol{h}_{m, k}^{\mathrm{T}} \boldsymbol{F}_{m} \boldsymbol{w}_{m, k}\right|^{2}}{\sum_{j \neq k} \frac{\rho \beta P}{K}\left|\boldsymbol{h}_{m, k}^{\mathrm{T}} \boldsymbol{F} \boldsymbol{w}_{m, k}\right|^{2}+\sigma_{m, k}^{2}} .
$$

The corresponding achievable rate is then

$$
r_{m, k}=\mathrm{lb}\left(1+\operatorname{SINR}_{m, k}\right),
$$

and the achievable sum rate of the group is calculated by

$$
R_{m}=\sum_{k=1}^{K} r_{m, k}=\sum_{k=1}^{K} \mathrm{lb}\left(1+\operatorname{SINR}_{m, k}\right) .
$$

During all of the $G$ time slots, the achievable sum rate of the $N$ user can be given by

$$
C=\sum_{m=1}^{G} R_{m}=\sum_{m=1}^{G} \sum_{k=1}^{K} r_{m, k} .
$$

We employ the precoding algorithms proposed in Ref.[8] in our simulation. For the analog precoder,

$$
\left[\boldsymbol{F}_{m}\right]_{i, j}=\frac{1}{\sqrt{M}} \mathrm{e}^{\mathrm{j} 2 \pi \phi_{i, j}},
$$

where $\phi_{i, j}$ is the phase angle of the channel element $\left[\boldsymbol{H}_{m}^{\mathrm{H}}\right]_{i, j}$ For the digital precoder, an equivalent channel $\tilde{\boldsymbol{H}}_{m}=\boldsymbol{H}_{m} \boldsymbol{F}_{m}$ is seen at the baseband. When zeroforcing(ZF) precoding is applied, the digital precoder is then given by

$$
\boldsymbol{W}_{m}=\tilde{\boldsymbol{H}}_{m}^{\mathrm{H}}\left(\tilde{\boldsymbol{H}}_{m} \tilde{\boldsymbol{H}}_{m}^{\mathrm{H}}\right)^{-1} \Lambda .
$$

The diagonal matrix $\boldsymbol{\Lambda}$ is designed to normalize the precoding matrix in order to meet the power constraint in Eq.(5).

It is evident that different partitions of users lead to different performance since the SINR of each user is jointly determined by the precoding and channels, not to mention that the precoders are specifically designed based on the channel sub-matrix. The achievable sum rate of the system can be substantially increased if we employ smart partitions. The problem can be represented as 


$$
\left(\boldsymbol{H}_{1}^{*}, \boldsymbol{H}_{2}^{*}, \cdots, \boldsymbol{H}_{G}^{*}\right)=\underset{\substack{\left.\boldsymbol{i}_{1}, \boldsymbol{H}_{2}, \cdots, \boldsymbol{H}_{G}\right\} \\ \text { a partition of } \boldsymbol{H}}}{\arg \max }\left\{\sum_{m=1}^{G} \sum_{k=1}^{K} \mathrm{lb}\left(1+S I N R_{m, k}\right)\right\} .
$$

\section{Proposed scheduling scheme}

It is NP-hard to exhaustively search for the optimal partition due to the sheer number of combinatorial grouping choices. On the other hand, random partitions lead to performance deterioration due to the strong correlation between channels in a satellite system. In this section, two heuristic scheduling algorithms are proposed to solve this problem. When there are a large number of users connected within the system that need to be grouped, the proposed algorithms employ recursive methods in order to quickly complete the task.

It has been observed that the benefits of hybrid precoding are in the power boost and interference cancellation of beamforming. When channels between different users exhibit a strong correlation, the interference cancelation becomes much more challenging, and the performance gain of precoding becomes non-existent. Therefore, we will divide these strongly correlated users into different slots in order to approach the optimal performance level, but with much lower computational complexity.

Based on the above principles, we start by searching for the pair of users who share the strongest channel coherence among all pairs of users, and then create two queues with each of the first pair of users as the head of a queue. This operation is then repeated for the remaining users, and each user is placed next in line in one of the two queues. After all users have been traversed, there are two subgroups. Recursively, we can develop more elaborate partitions. Theoretically, this algorithm is focused on canceling the globally strongest coherence, and thus it has a performance that is superior to other recursive algorithms. The corresponding time complexity is approximately

$$
O\left(M^{2} N^{2} \log N\right),
$$

where $M$ is the number of transmitting antennas and $N$ is the number of users.

If there are large numbers of connected users, we developed an alternate algorithm that has an almost linear time complexity instead of nearly square order complexity. If we consider the case of an arbitrary user, for instance, User 1, calculate the channel vector coherence coefficients between User 1 and other users, sort them into descending order, and separate the odd and even indices in order to balance the channel correlation of the odd and even subgroups. Then the corresponding time complexity is approximately

$$
O\left[N \log N\left(\log N+M^{2}\right)\right],
$$

where $M$ is the number of transmitting antennas and $N$ is the number of users.

We refer to Algorithms 1 and 2 as using the intergroup-coherence-balancing method and the globallystrongest-coherence-cancellation method respectively. The pseudocode for one single bisected stage is shown below.

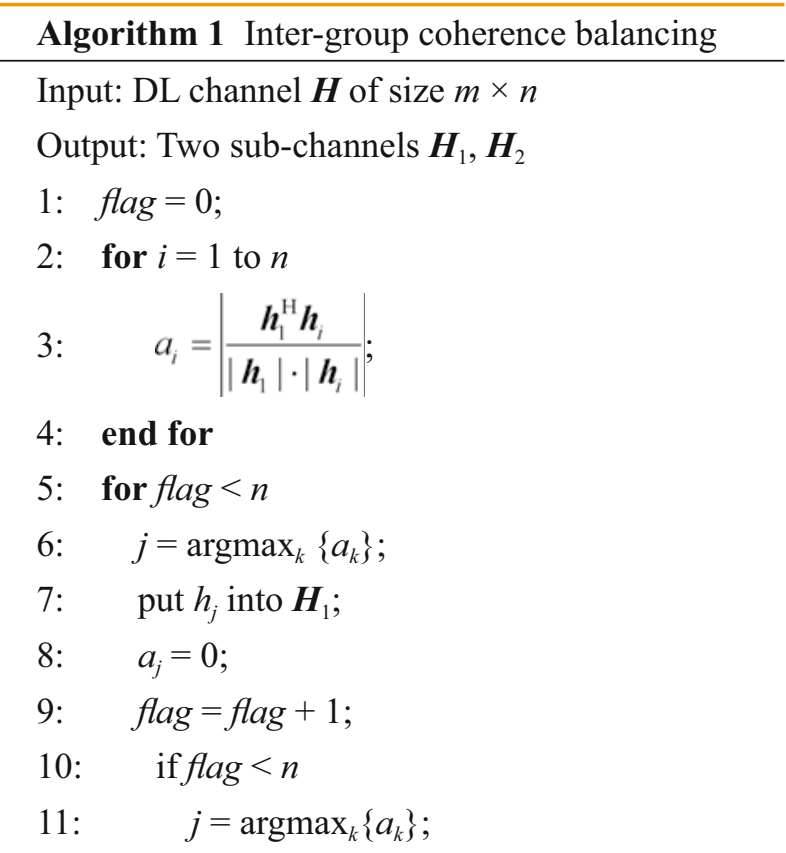


12: $\quad$ put $\boldsymbol{h}_{j}$ into $\boldsymbol{H}_{2}$;

13: $\quad a_{j}=0$;

14: $\quad$ flag $=$ flag +1 ;

15: end if

16: end for

Adopting the bisected methods, we divide one group of users into two sub-groups. Recursively doing the work on the sub-groups $\boldsymbol{H}_{1}, \boldsymbol{H}_{2}$, we can finally complete the scheduling.

Algorithm 2 is more considerate but has a higher degree of computational complexity. We start by finding the most correlated pair, and then separate them into two groups. This is completed for all remaining users.

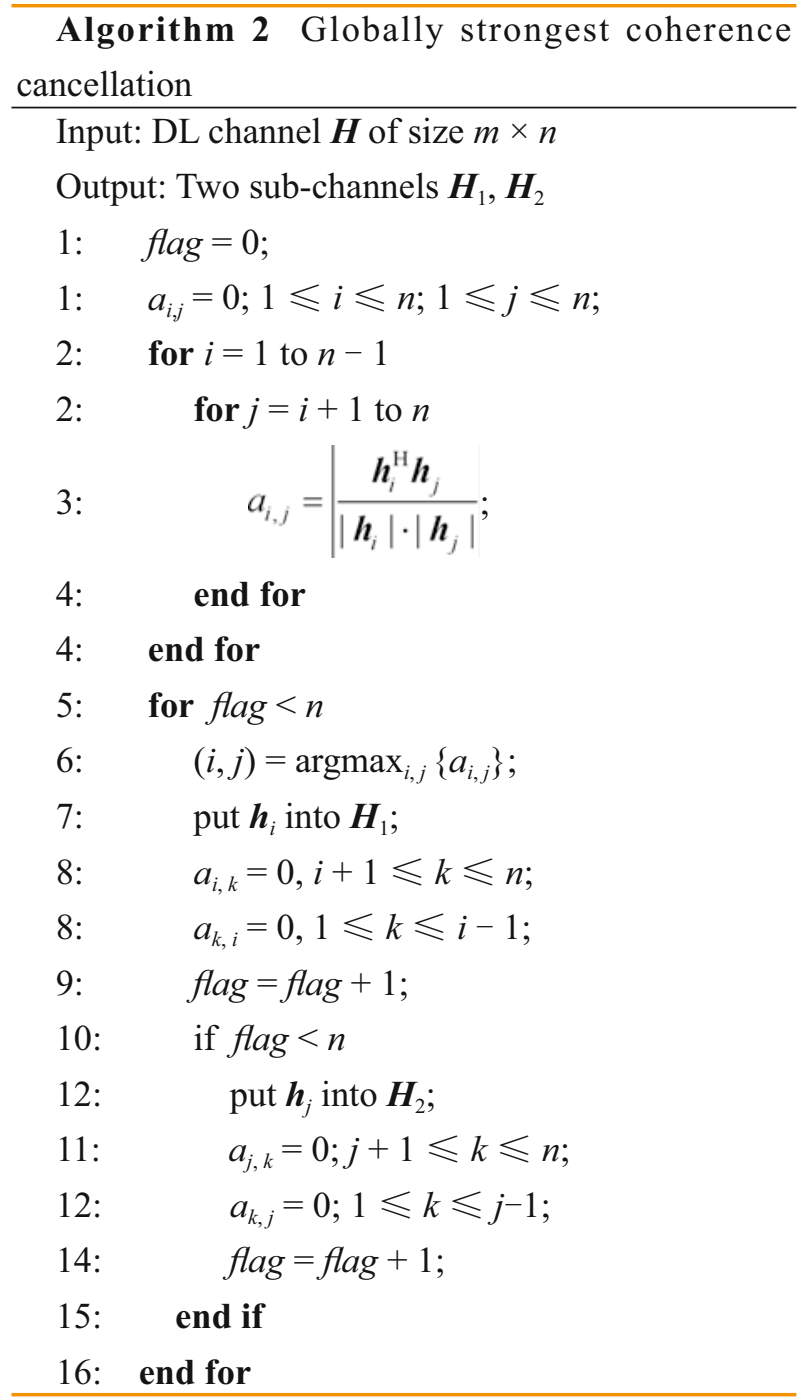

\section{Simulation results}

\subsection{Time complexity verification}

To verify the time complexity analyses, we change the scale of users to be grouped, and keep a record of the corresponding time for the simulated implementation of the two algorithms. The results shown in Figs.3 and 4 can be regarded as confirmation of the theoretical analyses. Algorithm 1 exhibits almost linear complexity while Algorithm 2 exhibits time complexity of nearly square order.

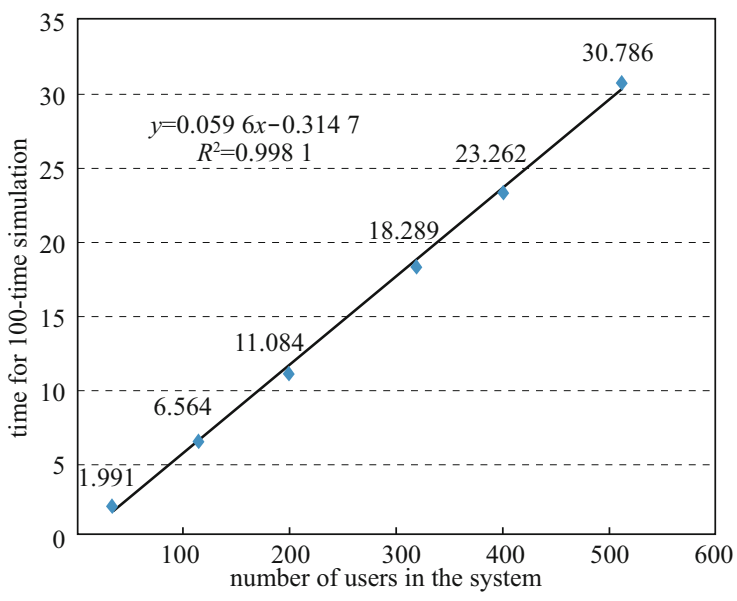

Figure 3 Algorithm 1 time complexity verification

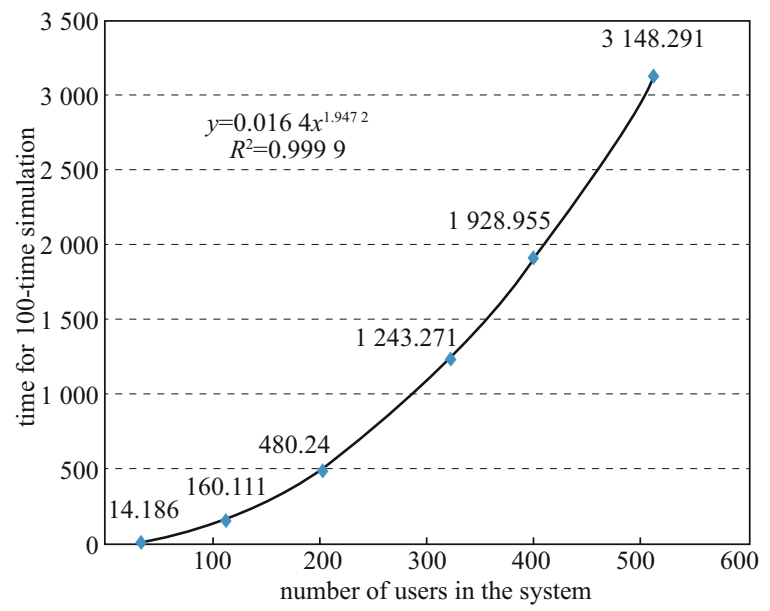

Figure 4 Algorithm 2 time complexity verification 


\subsection{Achievable sum rate comparison}

Noticing that Eq.(8) can be rewritten as

$$
\operatorname{SINR}_{m, k}=\frac{\left|\boldsymbol{h}_{m, k}^{\mathrm{T}} \boldsymbol{F}_{m} \boldsymbol{w}_{m, k}\right|^{2}}{\sum_{j \neq k}\left|\boldsymbol{h}_{m, k}^{\mathrm{T}} \boldsymbol{F} \boldsymbol{w}_{m, j}\right|^{2}+\frac{K \sigma_{m, k}^{2}}{\rho \beta P}},
$$

if we take a reasonable assumption $\sigma_{m, k}^{2}=\sigma^{2}$, we can define

$$
\eta=\frac{\rho \beta P}{K \sigma^{2}}
$$

$\eta$ can be considered as the received SNR (Signal-toNoise Ratio) without precoding. We adopt $\eta$ as the abscissa instead of the total transmitting SNR since it makes no difference.

In order to consider practical situation, we employ both directionally fixed and directionally adaptive beamforming scenarios in our simulation. In directionally fixed beamforming, the precoders are designed based on $K$ fixed directions, and remain unchanged during all of the $G$ time slots. The advantage of this scheme is that the implementation is low complexity because only one precoder setting is needed; however, the rate performance is poor due to the lack of channel state information in the design of the precoders. In directionally adaptive beamforming, targeted precoders are devised for each group that take into account the characteristics of the channels being used.

In Fig.5, the optimal solution obtained after exhaustive searching is compared with the proposed algorithms, where $N=8, G=4$, and $K=2$. For values of N larger than 10 , the simulation tends to collapse due to the astronomical number of pairs produced. This is why no optimal upper bound is displayed in the simulation results in this paper.

The simulation results are based on the following conditions: $N=256, G=8, K=32$, and 16 $\times 16$ URA. We simulated the random/optimized scheduling scheme with directionally fixed/adaptive ZF beamforming in order to investigate the performance of our proposed method. The rate- $\eta$ curves are shown and the probabilistic properties are investigated using CCDF (Complementary Cumulative Density Functions).

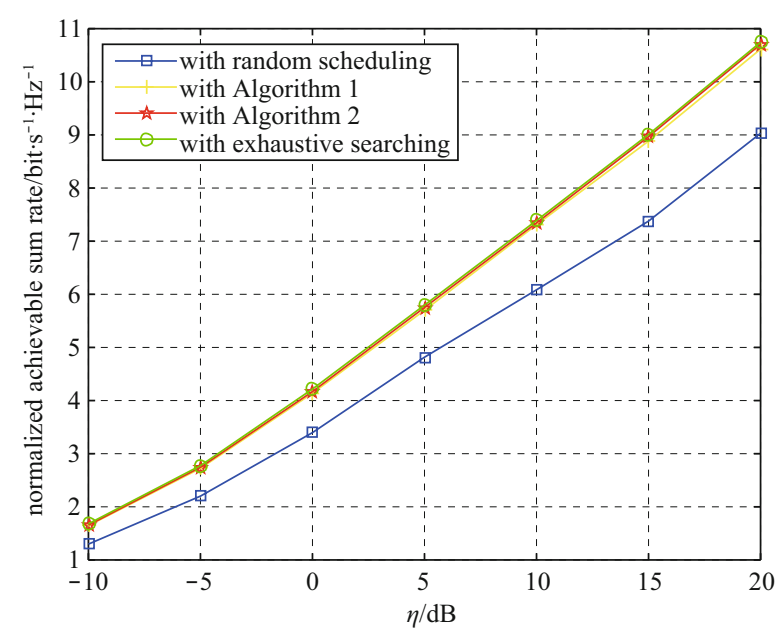

Figure 5 Capacity performance with ZF precoder in satellite systems

In the figure legends, $\mathrm{RD}$ is short for random scheduling, OP1 for optimized scheduling with Algorithm 1, OP2 for optimized scheduling with Algorithm 2, F for directionally fixed, $\mathrm{A}$ for directionally adaptive, and $\mathrm{BF}$ for beamforming.

We have three conclusions based on the simulation results as shown in Figs.6 and 7.

1) With directionally adaptive $B F$, the sum rate is much higher than that of fixed BF regardless of the scheduling scheme. This is due to the fact that the precoders in adaptive $\mathrm{BF}$ are designed based on the location of the users, while fixed BF adopts fixed precoders regardless of where the users are actually located.

2) With directionally adaptive BF, optimized scheduling is capable of increasing the sum rate when compared to the sum rate with random scheduling. This is due to the fact that the optimized scheduling algorithm ensures that there are no closely related 


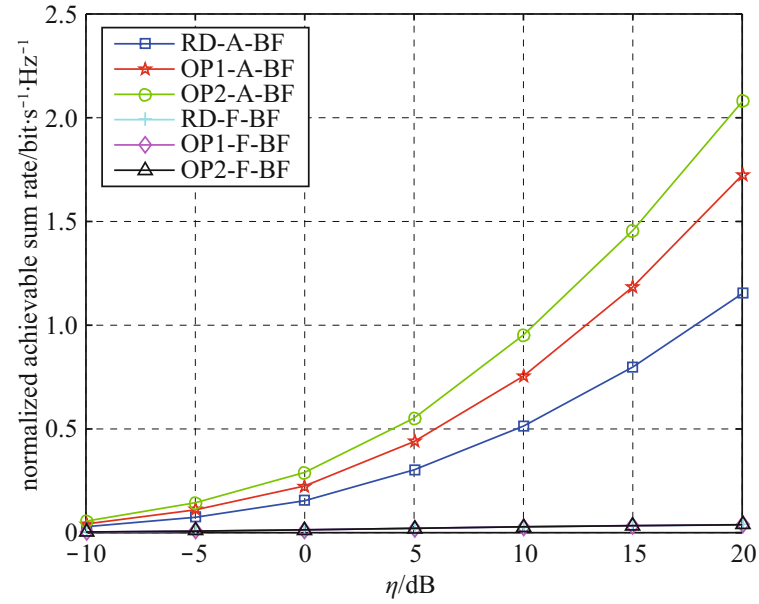

Figure 6 System sum rate with ZF precoder in satellite systems

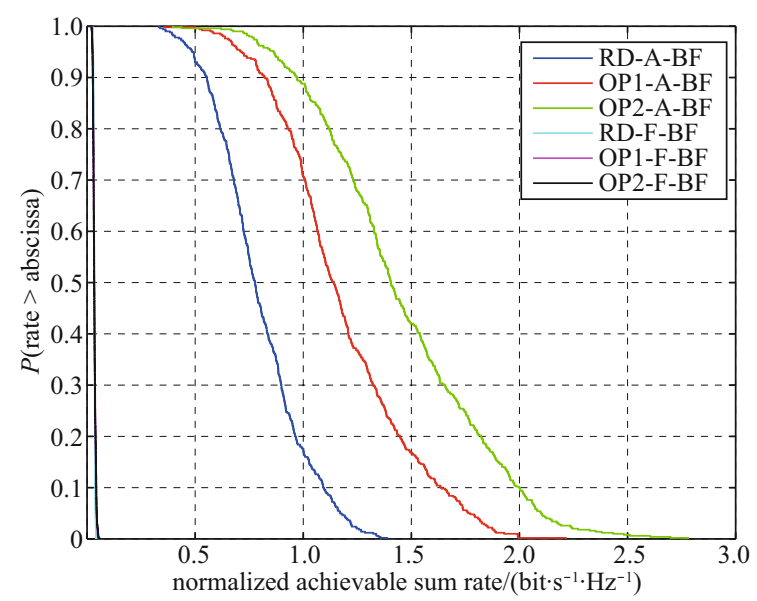

Figure 7 CCDF with ZF precoders in satellite systems $(\eta$ $=5 \mathrm{~dB})$

users in one group to deteriorate system performance. In addition, in adaptive $\mathrm{BF}$, the precoders are designed based on the locations of the users. Consequently, Algorithm 2 has a higher computational complexity although it also provides better performance than that of Algorithm 1.

3) With directionally fixed BF, optimized scheduling algorithm provides no benefit. Even though the scheduling algorithm separates related users into different groups, the fact that fixed BF uses fixed precoders means that the algorithm cannot benefit from this advantage. As a result, the sum rate remains virtually unchanged under the different scheduling schemes.

We also investigated the sum rate performance of our proposed scheduling algorithms with adaptive $\mathrm{BF}$ in land wireless communication systems, and the results are shown in Fig.8. In this case, the performance of Algorithm 2 is superior to that of Algorithm 1.

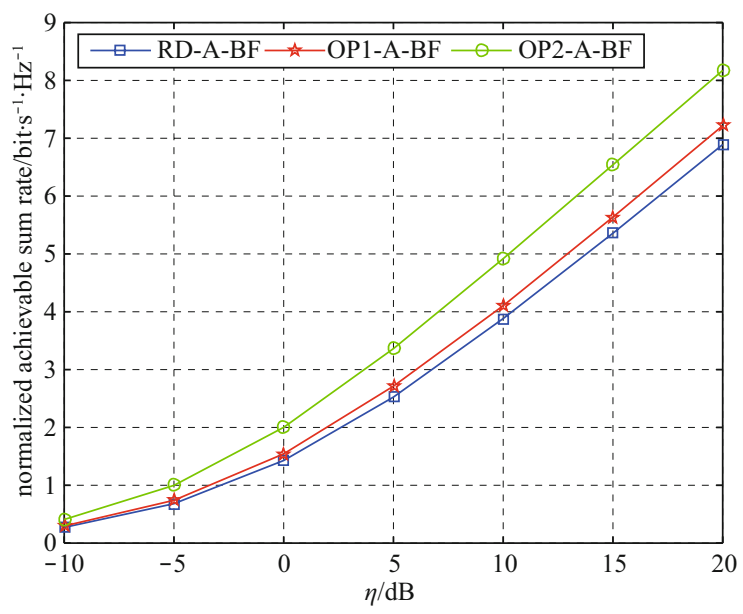

Figure 8 Adaptive BF with ZF precodomg in land wireless systems

Generally in satellite systems, there are a large number of users in the network, and resources are severely limited, such as time, frequency, hardware, power, etc. Under these conditions, the level of computational complexity in most cases determines the feasibility of a particular algorithm. Since Algorithm 1 requires only a linear sequence of vector correlation computations, it is more feasible. On the other hand, due to the strong correlations between satellite channels, if the simple cost of preventing the worst pairs from being aggregated in the same group can be accommodated, then the system sum rate can be considerably improved. 


\section{Conclusion}

In this paper, we proposed two optimized algorithms for scheduling, each with different computational complexities.

We have performed simulations of these algorithms in various scenarios and conclude that, if the scheduling algorithms that incorporate adaptive BF are adopted, then the achievable sum rate of the system can be considerably improved in terms of both the mean value and probability distribution. Algorithm 1 may be more preferable in satellite systems that have insufficient resources because it provides reasonable performance while having an extremely low computational complexity in contrast to Algorithm 2 when ZF precoding is adopted. We have further verified the feasibility of the proposed algorithms in land wireless systems. However, there is much potential improvement in designing an optimal scheduling scheme, especially when timefrequency-space resources must be dynamically and synergistically allocated. This is a topic for future research.

\section{References}

[1] YONG S K, CHONG C-C. An overview of multigigabit wireless through millimeter wave technology: potentials and technical challenges[J]. EURASIP journal on wireless communications and networking, 2006, 2007(1): 1-10.

[2] PI Z, KHAN F. An introduction to millimeter-wave mobile broadband systems[J]. IEEE communications magazine, 2011, 49(6): 101-107.

[3] PANAGOPOUlOS A D, ARAPOGLOU P D M, COTTIS P G. Satellite communications at $\mathrm{KU}, \mathrm{KA}$, and $\mathrm{V}$ bands: propagation impairments and mitigation techniques[J]. IEEE communications surveys \& tutorials, 2004, 6(3): 2-14.

[4] GONG S, WEI D, XUE X, et al. Study on the channel model and BER performance of single-polarization satellite-earth MIMO communication systems at ka band[J]. IEEE Transactions on antennas and propagation, 2014, 62(10): 5282-5297.

[5] KOUROGIORGAS C I, Panagopoulos A D. Multi satellite MIMO systems at Ka band and above: Outage capacity analysis using bivariate IG distribution[C]//The 8th IEEE European Conference on Antennas and Propagation (EuCAP), 2014: 1031-1034.

[6] BOJKOVIC Z S, BAKMAZ M R, BAKMAZ B M. Research challenges for $5 \mathrm{G}$ cellular architecture[C]//The 12th International Conference on Telecommunication in Modern Satellite, Cable and Broadcasting Services (TELSIKS), 2015: 215-222.

[7] ALKHATEEB A, AYACH O E, LEUS G, et al. Channel estimation and hybrid precoding for millimeter wave cellular systems[J]. IEEE journal of selected topics in signal processing, 2014, 8(5): 831-846.

[8] LIANG L, XU W, DONG X. Low-complexity hybrid precoding in massive multiuser MIMO systems[J]. IEEE wireless communication letters, 2014, 3(6): 653-656.

[9] ROH W, SEOL J Y, PARK J, et al. Millimeter-wave beamforming as an enabling technology for $5 \mathrm{G}$ cellular communications: theoretical feasibility and prototype results[J]. IEEE communications magazine, 2014, 52(2): 106-113.

[10] HSU K N, WANG C H, LEE Y Y, et al. Low complexity hybrid beamforming and precoding for $2 \mathrm{D}$ planar antenna array mmWave systems[C]// IEEE Workshop on Signal Processing Systems (SiPS), 2015: 1-6.

[11] NI W, DONG X. Hybrid block diagonalization for massive multiuser MIMO systems[J]. IEEE transactions on communications, 2015, 64(1): 201-211.

[12] ALKHATEEB A, LEUS G, HEATH R W. Limited feedback hybrid precoding for multi-user millimeter wave systems[J]. IEEE transactions on wireless communications, 2015, 14(11): 64816494.

[13] SOHRABI F, YU W. Hybrid digital and analog beamforming design for large-scale MIMO systems[C]//IEEE International Conference on Acoustics, Speech and Signal Processing (ICASSP), 2015: 29292933.

[14] BOUSSEMART V, BERIOLI M, ROSSETTO F. User scheduling for large multi-beam satellite MIMO systems[C]//Conference Record of the Forty Fifth Asilomar Conference on Signals, Systems and Computers (ASILOMAR), 2011: 1800-1804.

[15] AYACH O E, RAJAGOPAL S, ABU-SURRA S, et al. Spatially sparse precoding in millimeter wave MIMO systems[J]. IEEE transactions on wireless communications, 2014, 13(3): 14991513.

[16] SAYEED A, BRADY J. Beamspace MIMO for high-dimensional multiuser communication at millimeter-wave frequencies[C]//EEE Global Communications Conference (GLOBECOM), 2013: 36793684. 


\section{About the authors}

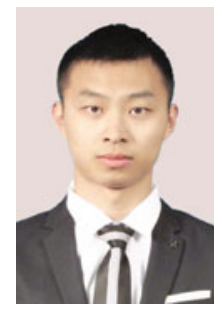

ZHOU Zhengyi was born in Hubei, China. He received a B.E. degree in electronic engineering from Tsinghua University, China, and is now a Ph.D. candidate in communications and information systems at Tsinghua University. His research interests include MIMO, precoding, and antenna systems and techniques. (Email: zhou zhengyi@126.com)

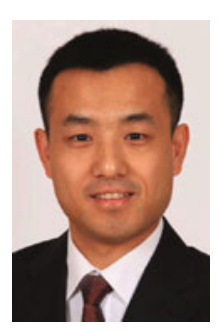

FENG Wei was born in Inner Mongolia, China. He received his B.S. and Ph.D. degrees in electronic engineering from Tsinghua University, China, and is now an assistant professor of electronic engineering at Tsinghua University. His research interests include distributed MIMO systems, coordinated resource allocation for wireless networks, and antenna array techniques for deep space communications. (Email: fengwei@tsinghua.edu.cn)

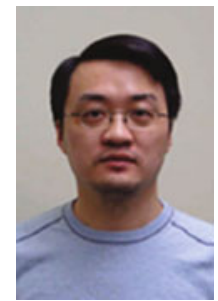

CHEN Yunfei received his B.E. and M.E. degrees in electronics engineering from Shanghai Jiao Tong University, Shanghai, China, in 1998 and 2001, respectively. He received his Ph.D. degree from the University of Alberta in 2006. He is currently working as an associate professor at the University of Warwick, U.K.. His research interests include wireless communications, cognitive radios, wireless relaying and energy harvesting.

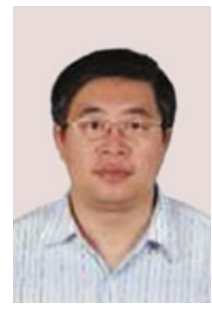

GE Ning [corresponding author] was born in Xi'an, China. He received his B.S. and Ph.D. degrees in electronic engineering from Tsinghua University, China, and is now a professor of electronic engineering at Tsinghua University. His research interests include communication ASIC design, short range wireless communications, and wireless communications. (Email: gening@tsinghua.edu.cn) 